\title{
Training In-Service Teachers to do Action Research in Educational Technology
}

\author{
Jayakrishnan Madathil Warriem, Sahana Murthy \\ IDP in Educational Technology \\ IIT Bombay, Mumbai, India \\ email:- \{jayakrishnan.m,sahanamurthy@iitb.ac.in\}
}

\author{
Sridhar Iyer \\ Dept. of Computer Science \& Engineering \\ IIT Bombay, India \\ sri@iitb.ac.in
}

\begin{abstract}
We describe the implementation and evaluation of a large-scale, distance mode, training workshop in Research Methods in Educational Technology (ET), for in-service engineering college teachers in India. The goal of the workshop was to prepare the teachers to conduct action research in their courses. The workshop consisted of 4 stages: i) pre-workshop assignment, ii) main workshop with two days of synchronous sessions on RM concepts, and a week in between of asynchronous hands-on activities, iii) 2-week post-workshop final assignment and iv) mentoring to conduct a research study. A total of 3896 teachers participated in the workshop, with $1141(29 \%)$ participants completing at least $50 \%$ of the assignments. To evaluate the effectiveness of the workshop, we conducted a study with pre-post research design. We found that learning gains from the pre- to the post-test were statistically significant. We administered a questionnaire to determine participants' learning perception and satisfaction from the workshop and found that a majority of responses $(>90 \%)$ were favourable.
\end{abstract}

Keywords- large-scale teacher training; research methods; teacher as researcher; action research;

\section{INTRODUCTION}

Many faculty members support the view that research enhances teaching [1]. Classroom action research in particular, is one form of integrating teaching and research. The Research Methods in Educational Technology (RMET) workshop, part of the "Teach 10 Thousand Teachers" (T10KT) initiative (under the Government of India's "National Mission in Education through ICT" [2]) is a step in training in-service teachers to perform action research. By the use of synchronous sessions, asynchronous activities and subsequent mentoring, this large scale (>3000 participants), distance-mode workshop trained in-service engineering college teachers to conduct an educational technology (ET) research study in their courses. In this paper, we describe the goals and implementation of the workshop, and discuss the research study we performed to evaluate the effectiveness of RMET workshop.

The workshop was organized in four stages, consisting of synchronous as well as asynchronous activities: i) a preworkshop stage in which participants individually worked on an assignment to propose a research idea on their practice, and submitted it on Moodle; ii) two, day-long synchronous sessions separated by week, during which they were introduced to formal concepts and practices of educational technology research, iii) a 2-week long set of assignments on conceptual understanding and application of research methods, and iv) a post-workshop mentoring stage for selected participants. The various stages of the workshop guided participants to progress from their initial proposal to a plan for a systematic research study. Participants worked on several hands-on activities, scaffolded by guidelines and templates such as the Idea Proposal Template (IPT) and the Study Planning Template (SPT). Selected participants underwent a 4-month long mentoring process during which they executed their research plan and conducted the study. Finally, participants who were successful in completing their study reported their findings as a research paper, which they submitted to the T4E 2013 conference.

We conducted a study to examine the effectiveness the workshop for large-scale training of in-service teachers to conduct ET research in their classes. Our research study used a single group pre-post design. Data were collected from multiple sources. The rate of participation was calculated from the number of participants who submitted various assignments at each stage of the workshop. To analyze learning gains, questions in the Idea Proposal Template were used as a pre-test to assess the level of application research methods knowledge, while questions in the Study Planning Template were used as the corresponding post-test. Responses to a survey questionnaire provided data on participants' perceptions of their own learning and satisfaction.

The workshop had a total of 3896 active participants, out of which different subsets participated in different activities. We found that 1141 (28.9\%) participants completed atleast $50 \%$ of the assignments, while $242(6.2 \%)$ among them completed all assignments. Our results showed that the learning gain was statistically significant $(Z=-12.4969$, $\mathrm{p}<0.001$ on a Wilcoxon Signed Rank test) with large effect size $(r=-0.566)$. In terms of participants' perception, $93.7 \%$ reported satisfaction with the workshop.

In the subsequent literature review section, we report related teacher training and faculty development programs, focusing on training in research methods and teacher action research. We provide details of the workshop implementation in section III and discuss the specific requirements and constraints which led to various choices for the design of the workshop. We describe the research methodology and instruments used to answer our research questions in section IV. We report the results of the various analyses and its subsequent discussion in sections $\mathrm{V}$ and VI respectively. 


\section{REVIEW OF LITERATURE}

Training programs and faculty development opportunities available to engineering college faculty include domain based training [3], as well as those that focus more on the pedagogical skills of the teachers such as SUCCEED [4], or 'Compulsory Lecturer Education' Norway [5]. There are also training programs or workshops on improving communication skills, or technology skills such as use of computers or multimedia techniques [6].

The programs which are committed in developing the teacher's role as a researcher are however few [6]. Of these, existing training programs on conducting research in education, include full-time courses [7], online courses [8] on social science research methods, or day-long workshops on individual research methodologies such as Case Method [9]. A limitation of these courses is that they remain isolated as academic programs, and there are fewer chances that the teacher will be able to practice educational research in their own context.

The idea of teacher becoming an action researcher has been suggested to improve the quality of teaching-learning process and prepare the teachers to think beyond their immediate practice [7]. Ponte suggested that action research should start with teachers' own situation and help them to engage in reflection through the gathered data [10]. This was operationalized in a program, which started with obtaining a general teaching idea from the teacher, explored it further through planning, implementing and evaluating the actions, and concluded with the writing up of the case study of the action research [11]. These steps were based on already established action research models of Elliot [12]. A structured reflective approach was suggested by O'Sullivan [13] for unqualified or under-qualified teachers, which built upon these models by the use of scaffolds.

Fincher and Tenenberg [14] mention three projects within engineering education - Bootstrapping, Rigorous Research in Engineering Education (RREE) and Institutes for Scholarship on Engineering Education (ISEE), which looked at conducting research in teaching-learning issues within teachers' own academic context. In the Bootstrapping project, a small number of Computer Science teachers were provided with initial workshop in Computer Science Education (CSEd) research to carry out action research within their workplace [14]. The RREE, given to a smaller cohort of engineering educators, asked more fundamental questions on how students learn engineering education [15]. The ISEE also focused on providing educational research knowledge to selected engineering educators and help them practice the research designs [15]. Though these programmes differed in delivery mechanisms, they shared a common idea of discipline based pedagogic enquiry to start the process of teacher action research.

In terms of discipline, the Bootstrapping project looked at the specific case of CSEd research while RREE and ISEE looked at engineering education in general [14][15]. Our Research Methods in ET workshop is more in line with the latter group of action research models within engineering education; however it differs from the two in terms of its large scale implementation. The second difference is in terms of mode of delivery. The popular modes of delivery of most of the training workshops discussed in previous paragraphs are either face to face or online. The T10KT initiative, which our RMET workshop is a part of, introduced a special mode within the distance education umbrella: multiple synchronous remote classrooms [16].

\section{REQUIREMENT AND TREATMENT}

\section{A. Requirement}

The major goal of the RMET workshop was to train engineering college teachers in planning, conducting, analyzing and reporting an educational technology research study. The learning objectives of the workshop were that the participants should be able to:

1. Gain familiarity with characteristics, types and examples of ET research.

2. Propose a well thought out idea and plan their ET research study.

3. Conduct the ET research study and report the findings.

The above learning objectives drove the workshop requirements and helped in deciding the overall sequencing. The objectives required instructional materials that would guide the participants in acquiring conceptual knowledge of research methods, as well as opportunities for practicing different aspects of ET research. In the workshop, we considered 'educational technology' to mean "a combination of processes and tools involved in addressing educational needs and problems" [17]. Hence guidelines and examples used in the workshop focused on the use of technology-based instructional strategies.

The implementation mode of T10KT initiative enabled the RMET workshop to be delivered to a large number of participants present in multiple synchronous remote classrooms located at various geographic locations within the country. This meant that all participants obtain simultaneous audio and video feeds of the sessions within their local classroom. The technology also enabled interaction between participants in different remote centers, and the central instructors who taught the sessions. Each synchronous remote classroom had a 'remote centre coordinator', who facilitated the sessions [16].

\section{B. Implementation}

Fig. 1 describes the implementation plan of RMET workshop connecting the goal, learning objectives and the activities. The entire implementation had four different stages: - Stage I: Pre-workshop stage, Stage II: Workshop Stage, Stage III: Post-Workshop and Stage IV: Mentorship Stage. These are now explained in detail. 


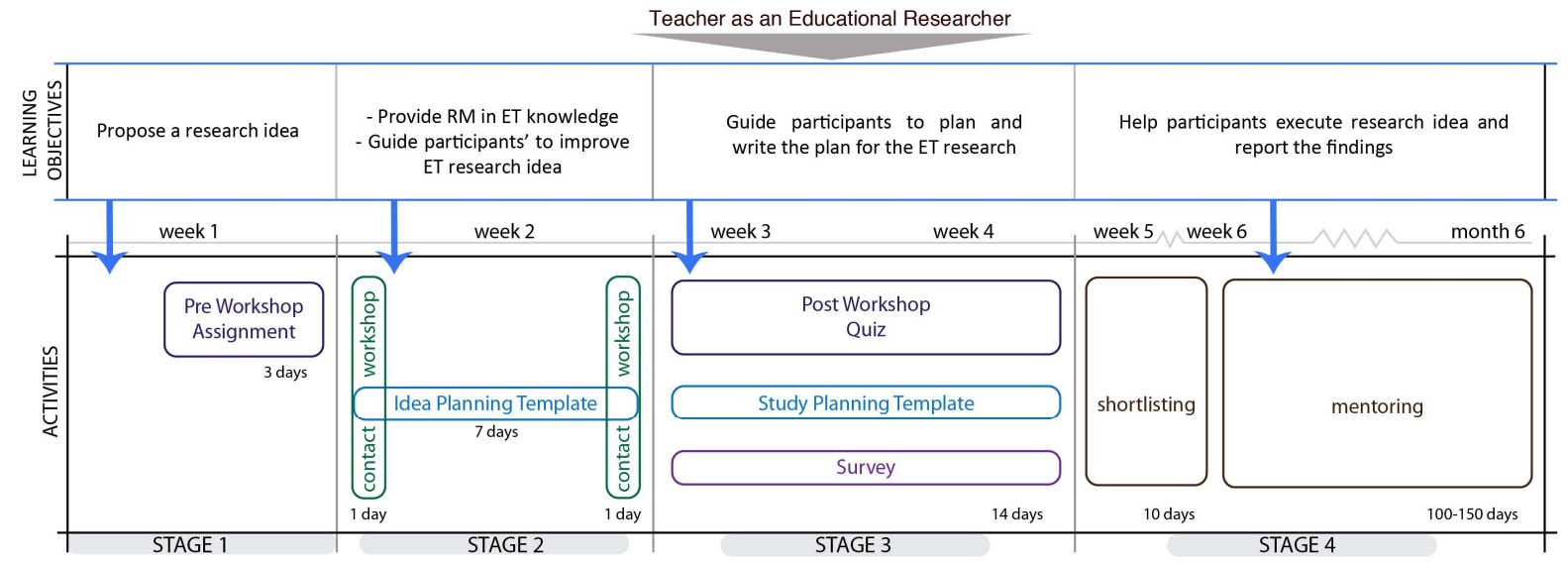

Figure 1: RMET Stagewise Implementation with Learning Objectives and Activities

\section{1) Stage I: Pre-Workshop}

In the pre-workshop stage, participants' proposed an initial research idea by answering a Pre-Workshop Assignment (PWA). The goal of the PWA was to get engineering college instructors to begin conceptualizing an ET study they could potentially conduct within the courses they taught. Participants were given three days to work on the assignment, which then had to be submitted on the Moodle page of the workshop.

\section{2) Stage II: Main Workshop}

The main workshop contained two days of synchronous sessions separated by a week of asynchronous activities. The sessions on the first day of synchronous sessions focussed on familiarizing the participants with the types of research studies conducted in educational technology. This gave them an opportunity to reflect on their initial research idea and its' positioning within this broad research space. The initial sessions introduced the participants to the evaluation criteria that are expected in a research paper Novelty, Positioning, Soundness of Procedure and Evidence [18], and explained how researchers need to pay attention to these criteria during the process of planning and conducting research.

In the next session, the participants were provided with two different published research papers and asked to analyze each of these on the basis of the four evaluation criteria. Apart from improving the critical analysis skills, this session also introduced them to current examples of ET research. This was followed by a session which introduced the Idea Proposal Template (IPT), containing a set of 11 questions designed to guide participants in various aspects of planning a research study.

One of the goals of the IPT was for participants to apply the evaluation criteria in their research study. The questions in the IPT guided participants in articulating their research idea, examining its novelty, reviewing related work, positioning their idea and devising a procedure to gather systematic evidence. Participants worked on an assignment based on the IPT during the week between the two synchronous days. Workshop instructors and TAs answered participants' questions related to the first three sessions and the assignment, via chat forums on Moodle.

The second synchronous day of the workshop (a week after the first) dealt with research methodologies. This included discussions on various research designs, choosing a sample, creation and validation of instruments, and ethics. The research design process was illustrated with practical examples of three existing experimental research studies of engineering college teachers. Each of these examples highlighted how novelty, positioning, soundness and evidence were addressed. The examples explained associated terminologies such as sampling, control and experimental groups, reliability, validity, statistical methods, and so on. This was followed up with a session on research ethics which dealt with topics like plagiarism, correct citations, summarizing and paraphrasing. These sessions included exercises so that participants could get enough time to practice. The final session also introduced the Study Planning Template (SPT), and a take-home assignment based on it.

\section{3) Stage III: Post-Workshop}

Along with the summative assessment of participant knowledge through a quiz, the post-workshop stage involved the completion of SPT based assignment. The purpose of this assignment was to give participants sufficient scaffolds to develop the research idea into a systematic study. Participants were asked to elaborate their research study by filling out the SPT. The SPT contained sections on setting up the problem, explaining the solution, and defending the solution, each of which had several prompts that the participants had to address in the context of their research study. Participants were given two weeks to complete the SPT assignment. These assignments were then evaluated using a grading rubric and a subset of them was shortlisted based on the quality of their SPT based assignment for further mentoring to conduct the study. 


\section{4) Stage IV: Mentorship}

The participants shortlisted in the post-workshop stage were assigned a mentor to guide them in planning, implementing and reporting their research study. The Ph.D. students in the Education Technology department at IIT Bombay were the mentors. Mentors were assigned based on the subject domain of the participant, the match of SPT idea to the mentor's research area and the research experience of the mentor. The discussions between the mentor and mentee mainly happened across e-mails and, if needed, telephonic conversations. Initially they provided the participants with an analysis of strength and weaknesses of the research idea and possible ways of refining it. Once the participants responded, they further provided guidance and clarifications at each and every stage of the research process. Along with this, the participants were provided with an opportunity to have a telephonic conference with workshop instructors.

\section{RESEARCH METHODOLOGY}

The research questions for this study are:

1. What was the rate of participation in the workshop?

2. What was the improvement in the participant's knowledge of ET research methods, both (a) measured and (b) perceived?

3. How satisfied were the participants with the workshop?

\section{A. Sample}

There were 5943 registered participants to the workshop; however, only 3896 out of them were involved at any stage within the workshop. The remaining never participated in any stage of the workshop, and they can be considered to have dropped their registration before the workshop began. All participants were engineering college instructors with varying years of experience. The workshop was announced in engineering colleges across the country. Participants selfselected to be part of the workshop. We obtained informed consent from the participants to use their responses to various assignments and their survey for this study.

To answer the research question related to the rate of participation within the workshop (RQ1), we examine the number of participants at key stages of the workshop involving assignment submissions. To answer the research question related to measured learning gains from the workshop (RQ2a), our study utilizes data from 242 participants who submitted assignments at each of the four stages. To answer questions related to the perception of learning (RQ2b), and satisfaction (RQ3), we utilize survey responses of 1286 participants who have given us consent to utilize the data.

\section{B. Instruments}

We have utilized three different instruments to collect data. These are:
1) Idea Proposal Template (IPT) based assignment. The IPT consisted of 11 questions, each of which targeted a specific criterion of novelty, positioning, soundness of procedure and evidence. For example, to address the positioning of research study there were questions on identifying existing journal articles and analyzing participants research idea in terms of the work in those papers. In order to address the criterion of soundness of procedure, the IPT contained questions related to the intervention in the study, such as: "What will the instructor do?" and "What will the students do?". The questions to address the criteria of evidence was "What evidence you will collect to show that your idea works".

2) Study Planning Template (SPT) based assignment. The various sections of the SPT contained questions that addressed different criteria in a research study. The template had three sections: setting up the problem (addressing novelty and positioning), explaining the solution (addressing soundness of procedure), and defending the solution (addressing soundness of procedure and evidence). Thus the quality of responses to questions in each SPT section provided a direct measure of participants learning from the workshop.

3) Survey Questionnaire - The participants were administered with a perception survey questionnaire which contained 18 questions on a five point Likert Scale (from Strongly Disagree to Strongly Agree). The questions relate to partcipants' perception of their learning from the workshop, and their overall satisfaction with the workshop. The survey was administered to the participants after the end of second synchronous day, via Moodle. An example of an item that addressed participants' perception of learning was, "As a result of the workshop, I feel confident of tackling educational technology research problems". Another question which trageted the satisfaction of participants' was : "Overall I am satisfied with the Workshop.'The survey also captured the open ended responses of the participants' perception of the elements of the workshop.

\section{Data Analysis Technique}

Literature provides terminologies like completion, persistence, retention or drop-out rates while looking at participation in similar large scale distance programs [19][20]. These terms look at continuous engagement of a participant within the program and impose a strict filter on the actual participation. We calculate both the strict 'persistence rate', that is, the number of participants completing all assignments, and a broad 'rate of participation', based on the number of participants submitting the four key assignments - PWA, IPT, SPT and Quiz. 
Participants' responses on the IPT and the SPT assignments were analyzed using a rubric. The rubric consisted of four dimensions of evaluation, corresponding to the criteria of novelty, positioning, soundness of procedure and evidence. Each dimension was evaluated on a 4-point scale: Very Low, Low, Medium and High, each of which had detailed descriptors relevant to the dimension being evaluated. The transition from the IPT to the SPT scores was analyzed to determine the measured learning gains from the workshop. The rubric was checked for validity and reliability before calculating the final scores. The inter-rater reliability was checked with three different graders and the kappa values were found to be more than 0.6 for each of the dimensions.

The survey responses were analyzed using frequency analysis of Likert Scale questions and content analysis of survey responses.

\section{RESULTS}

\section{A. Rate of Participation}

The rate of participation varied in each of the key assignments. A total of 3896 participants were involved across the various stages within the workshop. The PWA was attempted by 2215 participants $(56.9 \%)$ while 2311 participants (59.3\%) submitted IPT based assignments. Post contact workshop, 603 participants (15.4\%) attempted the quiz and 697 participants (17.9\%) submitted their SPT based assignments. The number of participants who submitted at least $50 \%$ of assignments was 1126 (28.9\%).

A stricter evaluation of the participation numbers is performed using the Persistence Rate, which is defined as the ratio of number of participants actively involved at all stages within the workshop to the total number of participants involved at any stage. Fig. 2 shows that 242 participants have submitted all four assignments within the workshop. Thus the persistence rate within the workshop was calculated to be $6.2 \%$.

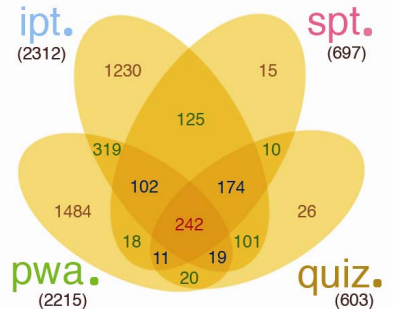

Fig 2: Participation numbers for each key submission

\section{B. Learning Gain from Workshop}

The learning gains from the workshop were calculated from: (a) the answers provided in the template based assignments (IPT and SPT) discussed in section IV, and (b) perception of learning gain of participants measured through the survey questionnaire. We calculate actual learning gains from the mean difference in performance between the SPT and the IPT-based assignment. For calculating learning gains we have used only the 242 participants who completed all the four activities. These were then graded using the template grading rubric and the scores of four criteria - Novelty, Positioning, Soundness of Procedure and Evidence, were analyzed. The scores of these rubrics were given from 0 to 3 corresponding to the four scales. Table 1 shows the overall score at both IPT and SPT stages.

As seen from the Table 1, from the IPT to SPT stage, there is a reduction in the number of participants at the 'Low' and 'Very Low' levels of assignment scores. The transition from the pre-test to the post-test scores is strong within the 'Medium' level where there is a visible increase by $28 \%$ (of total number of assignments). As data is not normally distributed, the most appropriate test was Wilcoxon signed-rank test. There was a significant transition of the scores from IPT (mean=2.9) to the SPT (mean=4.8) stage in terms of actual learning gain, $Z=-12.4969, \mathrm{p}<.001$, and the increase had a large effect size $(r=-0.566)$.

TABLE I. IMPROVEMENT OF SCORES ACROSS STAGES

\begin{tabular}{|c|c|c|}
\hline Scores & Pre-Test (IPT Score) & Post-Test (SPT Score) \\
\hline 0 (Very Low) & $15(6.19 \%)$ & $(0.83 \%)$ \\
\hline 1-4 (Low) & $221(91.32 \%)$ & $158 \quad(65.28 \%)$ \\
\hline 5-8 (Medium) & $6 \quad(2.48 \%)$ & $74 \quad(30.58 \%)$ \\
\hline 9-12 (High) & $0 \quad(0 \%)$ & $(3.3 \%)$ \\
\hline
\end{tabular}

The transition of the participants' mean scores from the IPT to the SPT stage across each of the dimensions is used for further analysis of actual learning gains. A state transition diagram across each performance level within a dimension is used for this purpose. The state transition diagram provides us with actual numbers along with empirical percentages of the transition.

1) Novelty: Fig. 3 shows the transition of Novelty scores from the IPT to the SPT stage. It can be seen that number of scorers at the Medium level increased from $2(0.8 \%$ of total participants) in pre-test to 48 (19.8\% of total participants) in post-test. The number of participants moving up from Very Low in pre-test to either Medium or High scores at post-test was 5 (2.1\% of total participants). Another 46 participants (19\% of total participants moved from Low level scores in pre-test to either Medium or High scores in post-test. Also none of participants' from the High or Medium pre-test scores moved down to Low or Very Low scores in the posttest. While performing the Wilcoxon signed-rank test, it is seen that the difference between mean IPT score (0.95) and mean SPT score (1.24) of the participants' is statistically significant, $Z=-7.48, p<.001$, and the increase was moderate. 


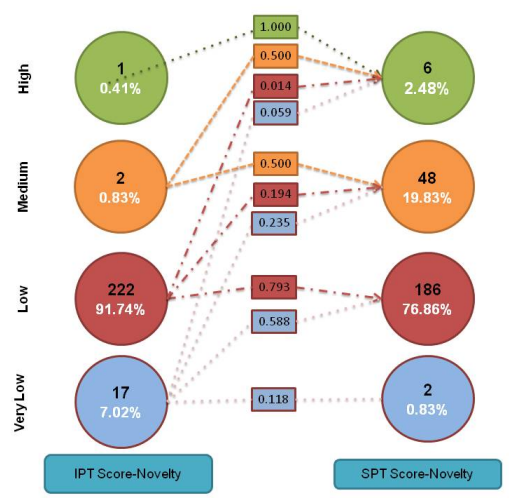

Figure 2. State transition from IPT to SPT for novelty dimension

2) Positioning: Fig. 4 shows the transition of participants' positioning scores from IPT to SPT stage. It is seen that the number of participants at the Medium level in post-test increased to 37 (15.3\% of total participants) from 2 $(0.8 \%$ of total participants) in pre-test. Similarly there is an increase of High scorers, with the numbers increasing from 0 in pre-test to $6(2.5 \%$ of total participants $)$ at post-test. The number of participants moving from Very Low and Low scores in pre-test to either High or Medium scores were 4 $(1.6 \%)$ and $37(15.3 \%)$ respectively. Once again, none of the High or Medium scorers at pre-test moved down to Low or Very Low scores in post-test. The difference between mean IPT score (0.92) and mean SPT score (1.19) of the participants' were found to be statistically significant, $Z=$ 7.252, $\mathrm{p}<0.001$, on performing Wilcoxon signed-rank test and the effect was moderate $(r=-0.329)$.

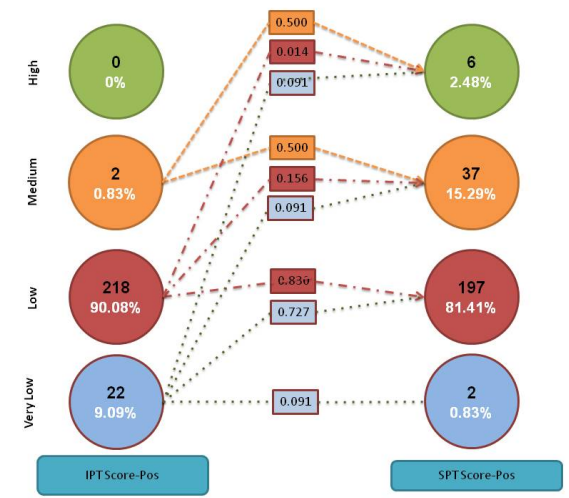

Figure 3. State Transition from IPT to SPT for positioning dimension

3) Soundness of Procedure: Figure 5 shows the transition of the soundness scores across the workshop. The number of participants having High score at post-test increased to $5(2.1 \%$ of total participants $)$ from 0 in the pretest. The Medium scorers at the post-test increased to 39 (16.1\% of total participants) from 1 at the pre-test stage. The number of particpants moving up from Very Low score in pre-test to Medium or High at post-test was 12 (5\% of total participants) while those moving from Low scores in pre- test were 31 ( $12.8 \%$ of total participants). Once again there are no drops from higher level while moving onto the SPT stage. The difference between particpants' mean IPT Score (0.39) and mean SPT Score (1.19) were found to be statistically significant while performing a Wilcoxon signed-rank test, $Z=-12.888, \mathrm{p}<0.001$, and the effect was large $(r=-0.586)$.

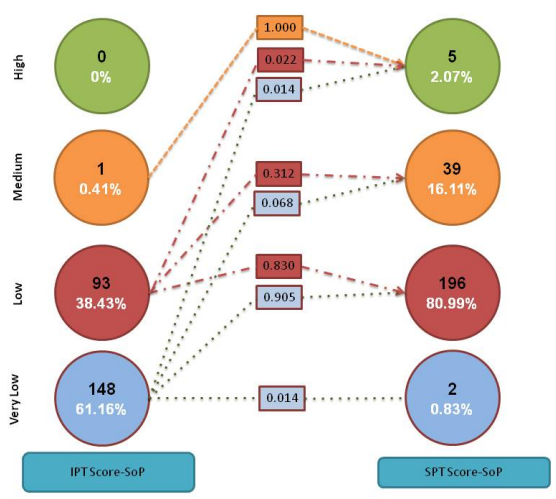

Figure 5: Transition with Soundness dimension

4) Evidence: Fig. 6 shows the state transition of evidence scores across the workshop. It can be seen that the number of High scorers at post-test increased to $4(1.7 \%)$ from 0 . The medium scorers also increased to $36(14.9 \%)$ from 3. The number of Very Low and Low scorers at pretest moving on to score High or Medium scores at post-test were $9(3.7 \%)$ and $30(12.4 \%)$ respectively. The Wilcoxon signed-rank test showed statistically significant difference between participants' mean IPT (0.62) and SPT scores (1.17), $\mathrm{Z}=-10.355$ and $\mathrm{p}<0.001$ with a moderate effect size $(\mathrm{r}=-0.471)$.

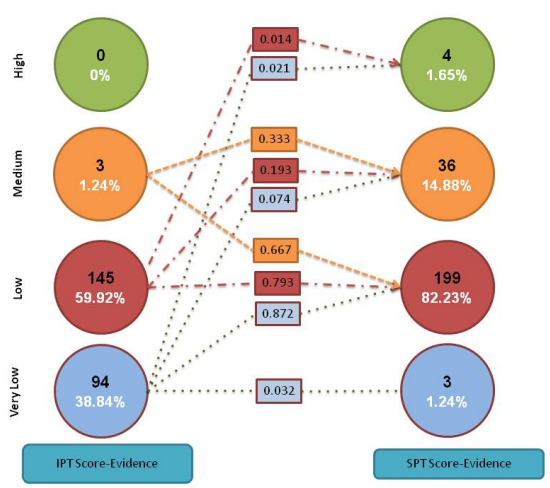

Figure 6: State Transition from IPT to SPT for Evidence dimension

\section{5) Perceptions of Learning Gain}

The perceived learning is captured through the responses of the participants to the survey question on learning within the workshop. $22 \%$ of the people strongly agreed and another $63.9 \%$ agreed on the point that they learnt from the workshop. Only $0.5 \%$ strongly disagreed to this, while another $1.3 \%$ showed general disagreement. 


\section{Overall Satisfaction}

The satisfaction of the participants' about the workshop mode was captured through the survey questionnaire and their open ended responses. It was seen that $60.1 \%$ of participants expressed satisfaction and another $33.6 \%$ expressed positive satisfaction to the workshop mode. Only $0.9 \%$ represented strong dissatisfaction while another $0.5 \%$ showed general dissatisfaction. This was further confirmed by analyzing the open ended responses of the participants. Most of the appreciated the various activities within the workshop like assignments, interactive sessions etc.

Comments like "[participants] were already practicing some ideas which is discussed in some sessions but with this workshop we realized how to make it as a research paper." is evidence that many were able to kick start the process of transition from practitioner to action-researcher. Many responded on the transfer of RM knowledge into other domains, as seen from the comment "[participants] got the information how to research, how to collect the data, how to analyze the data like, this workshop is very helpful". Majority of the participants also expressed immense satisfaction on getting introduced to new teaching-learning techniques which were used within the workshop.

The major dissatisfaction that was reported about the workshop by the participants was about the technical glitches in audio-video and lack of time. While majority commented about need for more time to submit IPT's, there were also a few who wanted to a greater duration of the contact workshop. The academic workload had hampered many is evident from this comment "time for paper analysis was insufficient, considering we were supposed to do it along with our official duties".

\section{DISCUSSION}

Table II summarizes the research questions, the various instruments that were used to answer the research questions and the key results for each research question.

TABLE II. IMPROVEMENT OF SCORES ACROSS STAGES

\begin{tabular}{|c|c|c|c|}
\hline $\begin{array}{l}\text { Research } \\
\text { Question }\end{array}$ & Instrument & Data collected & Result \\
\hline $\begin{array}{l}\text { What is the } \\
\text { rate of } \\
\text { participation? }\end{array}$ & $\begin{array}{c}\text { PWA, IPT } \\
\text { \& SPT } \\
\text { assignments } \\
\text { and Quiz }\end{array}$ & $\begin{array}{l}\text { Number of } \\
\text { people } \\
\text { attempting each. }\end{array}$ & $\begin{array}{l}28.9 \% \text { participants } \\
\text { submitted at least } 50 \% \\
\text { assignments, } 6.2 \% \\
\text { submitted all }\end{array}$ \\
\hline \multirow{2}{*}{$\begin{array}{l}\text { What is the } \\
\text { improvement } \\
\text { in participants' } \\
\text { ET research } \\
\text { methods } \\
\text { knowledge } \\
\text { after the } \\
\text { workshop? }\end{array}$} & $\begin{array}{c}\text { IPT and } \\
\text { SPT based } \\
\text { assignments }\end{array}$ & $\begin{array}{l}\text { Scores on } \\
\text { assignments } \\
\text { based on } \\
\text { templates, } \\
\text { across four } \\
\text { different } \\
\text { dimensions and } \\
\text { their total. }\end{array}$ & $\begin{array}{l}\text { The mean of IPT score } \\
\text { is } 2.9 \text { and mean of SPT } \\
\text { score is } 4.8 \text {. The } \\
\text { change was significant } \\
\text { with } Z=-12.4969, \mathrm{p}< \\
0.001 \text {, and effect was } \\
\text { large ( } \mathrm{r}=-0.566) \text {. }\end{array}$ \\
\hline & $\begin{array}{c}\text { Survey } \\
\text { Question }\end{array}$ & $\begin{array}{l}\text { Perception of } \\
\text { knowledge } \\
\text { gained }\end{array}$ & $\begin{array}{l}85.9 \% \text { participants } \\
\text { positively agreed that } \\
\text { they learnt from the } \\
\text { workshop. }\end{array}$ \\
\hline $\begin{array}{l}\text { Are the } \\
\text { participants } \\
\text { satisfied with } \\
\text { the workshop? }\end{array}$ & $\begin{array}{c}\text { Survey } \\
\text { Question }\end{array}$ & $\begin{array}{l}\text { Perception of } \\
\text { satisfaction and } \\
\text { open ended } \\
\text { responses }\end{array}$ & $\begin{array}{l}93.7 \% \text { of the } \\
\text { participants expressed } \\
\text { positive satisfaction. }\end{array}$ \\
\hline
\end{tabular}

Our first research question, "What is the "rate of participation" within the workshop activities?" was answered by looking at the number of participants submitting each of the assigned activity. We see that there was a high involvement during the initial stages of the workshop, with more than 2000 participants $(>55 \%)(2215$ for PWA and 2311 for IPT) submitting these. However the participation dropped as the workshop progressed. $28.9 \%$ actively participated by submitting atleast two of the four assignments and $17.9 \%$ completed the final assignment. The persistence rate, that is, those completing each and every assignment is low $(6.2 \%)$ and comparable to those reported in MOOCs [20].

The analysis of IPT and SPT based assignments provided us with scores for improvement in learning which was used to answer the second research question, "What is the improvement in participants' ET research methods knowledge as a result of the workshop?" This was backed by responses from survey on the perception of learning. The actual scores reflected a improvement of mean from 2.9 at IPT stage to 4.8 at SPT stage, Wilcoxon signed-rank test performed on the results showed that the difference in mean scores between IPT and SPT was significant with $Z=-$ $12.4969, \mathrm{p}<0.001$ with a large effect size $(\mathrm{r}=-0.566)$. On further analyzing the scores within each dimension we found that the Soundness of Procedure showed largest effect size $(\mathrm{r}=-0.586)$ followed by Evidence $(\mathrm{r}=-0.471)$. The other two dimensions had only moderate effect sizes even though the difference in scores was found to be statistically significant. Also for 3 out of 4 dimensions, none of the participants SPT (post-test) score was less than their IPT (pre-test) score, which is indicative of the knowledge gained within the workshop. The survey responses show that $85.93 \%$ of the participants either strongly agreed or agreed that **, consistent with the learning gain results from the pre-post tests.

The third research question "Are the participants satisfied with the workshop format?" was answered by analyzing the survey questionnaire and open ended responses. $93.71 \%$ of the participants expressed satisfaction with the workshop. However many had indicated the lack of time due to academic workload and technology failure as a serious deterrent to completion of the assignments and workshop satisfaction. Both these parameters were beyond our control within the current workshop. A possible way of tackling the first concern is by organizing the workshop at a time when the teachers have least academic workload.

Post workshop, the mentorship stage was designed to scaffold the teacher experiences further to convert the research ideas into a research paper to be submitted to the IEEE Conference on Technology for Education T4E 2013. There were a total of 10 submissions from the mentee participants and 4 from the non-mentored RMET participants. 9 out of the 10 mentee papers were accepted with 2 being accepted in their submitted category. None of the non-mentees papers got accepted for the final conference. These acceptance rates indicate the critical role played by mentorship process within the entire model. 
As suggested in [13], workshop model with the IPT \& SPT assignments and the mentorship process is aimed at providing a necessary scaffold to the novice ET action researcher. Participants' appreciation of workshop process and activities indicates that scaffolding at appropriate points was effective in helping practitioners engage in action research. Positive results of learning gains and participants' perceptions of learning (RQ2) indicate that our RMET workshop is a good step in addressing the gap of ET research methods knowledge for engineering instructors, as identified in [11].

A major limitation of this study is that we have not been able to get in touch with dropped out participants to know about their workshop experience. Hence, as indicated, the results of survey reflect the perception about people who already had a buy-in to the workshop.

\section{CONCLUSION}

In this paper we reported the implementation of a large scale workshop designed to train engineering college instructors in conducting research in their own practice. The workshop gives participants an exposure to such research, starting from familiarity with current studies, to conceptual knowledge of research methodologies and opportunities to apply that knowledge. We evaluated the effectiveness of the workshop from multiple perspectives: participants' rate of participation, learning gains, perceptions of learning, and satisfaction and found positive results overall for learning gains and satisfaction, but a low rate of participation. The study provides us with a list of useful tools, in terms of templates and activities, to execute similar workshops in the future.

\section{ACKNOWLEDGMENT}

The authors thank the entire research scholars at the IDP in Educational Technology department in IIT Bombay for their continuous support throughout the research experiment. The study was conducted on a programme run under National Mission on Education through ICT (NME-ICT) funded by the MHRD, Government of India.

\section{REFERENCES}

[1] R. Neumann, "Perceptions of the Research-teaching nexus: A Framework for Analysis," Higher Education, Vol. 23, 1992, pp. 159171

[2] "MHRD Workshops," 2009. [Online]. Available: http://www.it.iitb.ac.in/nmeict/pages/mhrdworkshops.jsp. [Accessed 6 May 2013].

[3] L. D. Camblin Jr. and J. A. Steger, "Rethinking Faculty Development,"Higher Education, vol. 39(1), January 2000, pp. 1-18.
[4] R. Brent and R. M. Felder, "A Model for Engineering Faculty Development,"International Journal for Engineering Education,Vol. 19(2), 2003, pp. 234-240.

[5] P. Trowler and R. Bamber, "Compulsory Higher Education Teacher Training: Joined-up policies, institutional architectures and enhancement cultures," vol. 10(2), 2005, 79-93.

[6] J. L. Kincheloe, "Teachers as Researchers: Qualitative Inquiry as a Path to Empowerment," New York: RouteledgeFarmer, 2003.

[7] R. Deem, "The Future of Educational Research in the Context of the Social Sciences: A Special Case?," British Journal of Educational Studies, vol. 44(2), June 1996, pp. 143-158.

[8] J. H. Herman, "Faculty Development Programs: The Frequency and Variety of Professional Development Programs available to Online Instructors," Journal of Asynchronous Learning Networks, vol. 16(5), 2012, pp.87-106.

[9] K. K. Merseth,"Cases and case methods in teacher education." Handbook of research on teacher education, 1996, pp. 722-744.

[10] P. Ponte, "How Teachers Become Action Researchers and How Teacher Educators Become Their Facilitators,", Educational Action Research, vol . 10(3), November 2002, pp 399-422

[11] P. Ponte, J. Ax, D. Beijaard and T. Wubbels, "Teachers' development of professional knowledge through action research and the facilitation of this by teacher educators," Teaching and Teacher Education, vol. 20, 2004, pp. 571-588.

[12] J. Elliott, "Action research for educational change," Open University Press, 1991.

[13] M. C. O'Sullivan, "Action research and the transfer of reflective approaches to in-service education and training (INSET) for unqualified and underqualified primary teachers in Namibia," Teaching and Teacher Education, vol. 18, no. 5, 2002, pp. 523-539.

[14] S. Fincher and J. Tenenberg, " Using Theory to Inform CapacityBuilding: Bootstrapping Communities of Practice in Computer Science Education Research," Journal of Engineering Education, October 2006, pp 1-13

[15] R. A. Stevler and K. A. Smith, "Conducting Rigorous Research in Engineering Education," Journal of Engineering Education, vol. , July 2006, pp 103-105.

[16] J. M. Warriem, S. Murthy and S. Iyer, "A model for Active learning in Synchronous Remote Classrooms: Evidence from a Large-Scale Implementation,", Proc. 21st International Conference on Computers in Education (ICCE '13), 2013, unpublished.

[17] M. D. Roblyer, "Integrating Educational Technology into Teaching" 4th ed., Prentice Hall, 2000.

[18] A. J. Smith, "The task of the referee," IEEE Computer, vol. 23, no. 4, April 1990, pp. 65-71.

[19] Y. Levy, "Comparing dropouts and persistence in e-learning courses," Computers \& Education, vol. 48, no. 2, February 2007, pp. 185-204

[20] M. Nicholas, "Student perceptions of support services and the influence of targeted interventions on retention in distance education," Distance Education, vol. 31, no. 1, 2010, pp. 93-113.

[21] K. Jordan, "MOOC Project," 2011 [Online]. Available: www.katyjordan.com/MOOCProject.html. [Accessed 5 May 2013]. 